OPEN ACCESS

Edited by:

Qingxiang Meng,

Hohai University, China

Reviewed by:

Muhammad Mubashir Bhatti, Shandong University of Science and

Technology, China

Zhang Ji,

East China Jiaotong University, China

*Correspondence:

Hongyang Sun

tju207@163.com

Specialty section:

This article was submitted to

Interdisciplinary Physics,

a section of the journal

Frontiers in Physics

Received: 08 September 2021 Accepted: 28 September 2021 Published: 15 November 2021

Citation:

Jin L, Sun H, Wang S and Zhou Z (2021) A Series Solution for $2 D$

Scattering of Cylindrical SH-Waves by Surrounding Loose Rock Zone of

Underground Tunnel Lining.

Front. Phys. 9:772823.

doi: 10.3389/fphy.2021.772823

\section{A Series Solution for 2D Scattering of Cylindrical SH-Waves by Surrounding Loose Rock Zone of Underground Tunnel Lining}

\author{
Liguo Jin ${ }^{1}$, Hongyang Sun ${ }^{2 *}$, Shengnian Wang $^{3}$ and Zhenghua Zhou ${ }^{4,3}$ \\ ${ }^{1}$ Institute of Geophysics, China Earthquake Administration, Beijing, China, ${ }^{2}$ Department of Civil Engineering and Architecture, \\ Yantai Vocational College, Yantai, China, ${ }^{3}$ College of Transportation Engineering, Nanjing Tech University, Nanjing, China, \\ ${ }^{4}$ Institute of Engineering Mechanics, Nanjing Tech University, Nanjing, China
}

This paper presents a closed-form series solution of cylindrical SH-wave scattering by the surrounding loose rock zone of underground tunnel lining in a uniform half-space based on the wave function expansion method and the mirror image method. The correctness of the series solution is verified through residual convergence and comparison with the published results. The influence of the frequency of the incident cylindrical SH-wave, the distance between the wave source and the lining, the lining buried depth, and the properties of the surrounding loose rock zone on the dynamic stress concentration of the tunnel lining is investigated. The results show that the incident wave with high frequency always makes the dynamic stress concentration of the tunnel lining obvious. With the increase of the distance between the wave source and the tunnel lining, the stress around the tunnel lining decreases, but the dynamic stress concentration factor around the tunnel lining does not decrease significantly but occasionally increases. The ground surface has a great influence on the stress concentration of the tunnel lining. The amplitude of the stress concentration factor of tunnel lining is highly related to the shear wave velocity of the surrounding loose rock zone. When the property of the surrounding rock (shear wave velocity) changes more, the amplitude of the stress concentration factor is larger, that is, the stress concentration is more significant.

Keywords: Underground tunnel, Lining, SH-wave, Analytic solution, Wave function expansion method

\section{INTRODUCTION}

The scattering of elastic waves by an underground cavity (or local topography) is one of the hot research topics in the fields of earthquake engineering, seismology, and geophysics due to its particular significance in seismic risk assessment, seismic microzonation, and the design of important facilities. When the seismic wave encounters a cavity (or local topography) during its propagation, it will produce a strong scattering effect, which in turn will affect the ground motion near the cavity (or local topography). The method of solving the problem of wave scattering can be divided into two kinds of methods: numerical method and analytical method. Numerical methods mainly include the finite difference method (FDM), finite element method (FEM), and boundary element method (BEM); the analytical methods mainly refer to wave function expansion methods. The numerical method can be applied to the cavity (or local topography) of any shape and various 
site conditions and is more suitable for handling actual engineering problems. The analytical method is still necessary to solve some special regular cavity (or local topography) and boundary conditions. Although the analytical method is only suitable for relatively simple and regular models, it has an advantage over the numerical method in revealing the essence of the problem, and it can also verify the accuracy of the numerical method.

For plane waves, beginning with the pioneering work of Trifunac [1, 2] on ground motion around a semi-circular alluvial valley and a semi-circular canyon embedded in a homogeneous isotropic half-space, several research works have been carried out on this topic both analytically and numerically. For the underground tunnel lining, the current closed-form analytical solutions are Refs. [3, 4]. For canyon topography, the present analytical solutions are Taur et al. [5], Gao et al. [6], Zhang et al. [7], Jin et al. [8-10], and Lee et al. [11, 12]. In addition, various numerical methods mainly include the finite difference method [13, 14], the improved Bouchon-Campillo method [15], the boundary integral equation method [16, 17], the null-field boundary integral equation method [18], the weighted residual method [19-21], and the boundary element method [22-29]. These research works have been widely reviewed by many scholars such as Sanchez-Sesma et al. [30], Liu et al. [31], Gao et al. [6], and Bhatti and $\mathrm{Lu}[39,40]$.

For cylindrical waves, Liang et al. [32] studied the scattering of cylindrical $\mathrm{SH}$-waves by underground lining caverns using the mirror image method. Li [33] investigated the numerical solution of the cylindrical SH-wave scattering by a circular hole. Zhang [34] studied the scattering of cylindrical waves by underground circular sandwiched areas and lining caverns in the half-space by using a special boundary integral equation method. Xu et al. [35] investigated the diffraction of Rayleigh waves around a circular cavity in the poroelastic half-space by using an indirect boundary integral equation method based on Biot's two-phase medium theory.

This paper notices that the above-mentioned studies are mostly aimed at plane $\mathrm{SH}$-waves and do not consider the impact of cylindrical SH-waves on the surrounding rock zone (i.e., the generation of loose circles) generated during cavity blasting and excavation. Therefore, this paper establishes an analytical model for the scattering of cylindrical $\mathrm{SH}$-waves by loose rock circles around the underground lining cavern embedded in a $2 \mathrm{D}$ homogenous half-space and uses the wave function expansion method to obtain the series solution of scattering.

In the next section, the methodology is presented, followed by the verification through residual convergence and comparison with the published results of Liang et al [32]. Then, the results in the frequency domain are presented, and the anti-plane tunnel responses are discussed. Finally, the main findings and the conclusions are summarized.

\section{METHODOLOGY}

\section{Analytical Model}

As shown in Figure 1A, the inner and outer radii of the circular lined tunnel are $a$ and $b(b=0.9 a)$, respectively. The surrounding
A The model of 2D scattering of cylindrical SH-waves by surrounding loose rock zone of underground tunnel lining

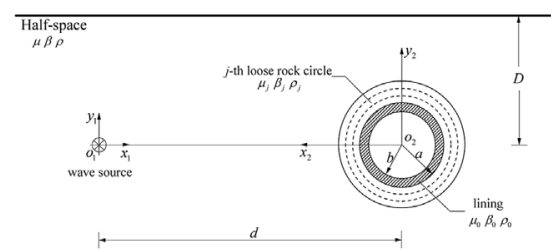

B The mirror image of the model

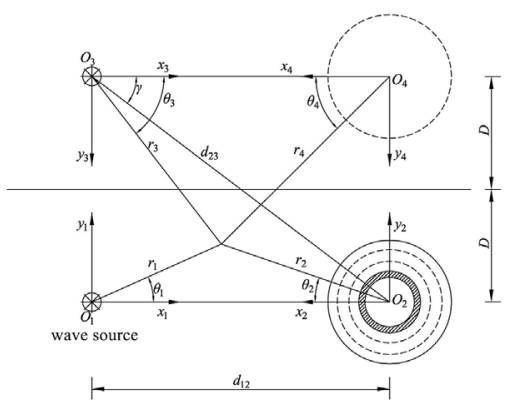

CNormalization of dynamic stresses on lining inner and outer surfaces

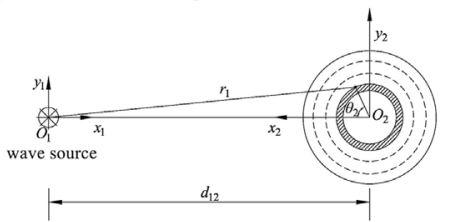

FIGURE 1 | Model information.

loose rock zone is divided into $j$ layers, and the radius of each layer is $c_{1}, c_{2}, c_{3}, c_{j} \ldots$ from inside to outside. The burial depth of the circular lined tunnel is $D$. The half-space, the surrounding loose rock zone, and the lining are assumed to be linearly elastic, uniform, and isotropic media. The half-space is marked with shear wave velocity $\beta$, mass density $\rho$, and shear modulus $\mu$; the $j$ th surrounding loose rock is marked with shear wave velocity $\beta_{j}$, mass density $\rho_{j}$, and shear modulus $\mu_{j}$; the lining is marked with shear wave velocity $\beta_{0}$, mass density $\rho_{0}$, and shear modulus $\mu_{0}$. The center of the wave source and the underground cavity is located at the same depth, and the distance between them is $D_{12}$.

\section{Governing Equations and Boundary Conditions}

The cylindrical $\mathrm{SH}$-wave with unit amplitude generated by the wave source at point $O_{1}$ can be expressed as

$$
W_{i 1}\left(r_{1}, \theta_{1}\right)=H_{0}^{(1)}\left(k r_{1}\right) e^{-i \omega t},
$$

where $k=\omega / \beta$ is the wavenumber of the SH-wave in the halfspace, $\omega$ is the circle frequency of the incident wave, and $i=\sqrt{-1}$ represents the imaginary unit. $e^{-i \omega t}$ is the time factor, and it will be omitted in the following mathematical derivation. Considering 
the axisymmetric properties of the wave source, the Hankel function is of order 0 .

The reflected wave will be generated when the incident cylindrical $\mathrm{SH}$-wave propagates to the ground surface, and a scattered $\mathrm{SH}$-wave will be generated when the incident cylindrical $\mathrm{SH}$-wave encounters a cavity. Then, the total wave motion field in the half-space is the superposition of the incident wave, reflected wave, and scattered wave. Meanwhile, the wave will also diffract into the lining and surrounding loose rock, and all these waves must satisfy the following wave equation:

$$
\frac{\partial^{2} w}{\partial r^{2}}+\frac{1}{r} \frac{\partial w}{\partial r}+\frac{1}{r^{2}} \frac{\partial^{2} w}{\partial \theta^{2}}=\frac{1}{\beta^{2}} \frac{\partial^{2} w}{\partial t^{2}}
$$

To satisfy the zero-stress boundary condition of the half-space surface, the mirror image method [3] is used to solve the problem. As shown in Figure 1B, assume that there is another cylindrical wave source and cavity with the same surrounding loose rock zone in the half-space with the surface as its axis of symmetry. The mirror incident $\mathrm{SH}$-wave source $W_{r 3}\left(r_{3}, \theta_{3}\right)$ is

$$
W_{r 3}\left(r_{3}, \theta_{3}\right)=H_{0}^{(1)}\left(k r_{3}\right) \text {. }
$$

The scattered wavefield corresponding to the two cavities can be expressed as

$$
\begin{aligned}
& W_{s 2}\left(r_{2}, \theta_{2}\right)=\sum_{n_{2}=0}^{+\infty} H_{n_{2}}^{(1)}\left(k r_{2}\right)\left(A_{s 2} \cos n_{2} \theta_{2}+B_{s 2} \sin n_{2} \theta_{2}\right), \\
& W_{s 4}\left(r_{4}, \theta_{4}\right)=\sum_{n_{4}=0}^{+\infty} H_{n_{4}}^{(1)}\left(k r_{4}\right)\left(A_{s 4} \cos n_{4} \theta_{4}+B_{s 4} \sin n_{4} \theta_{4}\right) .
\end{aligned}
$$

In a physical sense, Eq. 5 represents the wave propagating outward from $\mathrm{O}_{2}$ and $\mathrm{O}_{4}$ in the whole space and satisfies the wave equation (3) and Sommerfeld radiation conditions. $A_{s 2}, B_{s 2}, A_{s 4}$, and $B_{s 4}$ are the undetermined complex constants, and $A_{s 2}=A_{s 4}$, $B_{s 2}=B_{s 4}$. The wave motion in the whole space is

$$
W=W_{i 1}\left(r_{1}, \theta_{1}\right)+W_{s 2}\left(r_{2}, \theta_{2}\right)+W_{r 3}\left(r_{3}, \theta_{3}\right)+W_{s 4}\left(r_{4}, \theta_{4}\right) .
$$

The expression of the scattered field generated in the lining in the polar coordinate system $\left(r_{2}, \theta_{2}\right)$ can be written as

$$
\begin{aligned}
& W_{01}\left(r_{2}, \theta_{2}\right)=\sum_{n_{2}=0}^{+\infty} H_{n_{2}}^{(1)}\left(k_{1} r_{2}\right)\left(C_{01} \cos n_{2} \theta_{2}+D_{01} \sin n_{2} \theta_{2}\right), \\
& W_{02}\left(r_{2}, \theta_{2}\right)=\sum_{n_{2}=0}^{+\infty} J_{n_{2}}\left(k_{1} r_{2}\right)\left(C_{02} \cos n_{2} \theta_{2}+D_{02} \sin n_{2} \theta_{2}\right) .
\end{aligned}
$$

Here, $W_{01}\left(r_{2}, \theta_{2}\right)$ is the wave propagating outward from $\mathrm{O}_{2}$ in the lining and $W_{02}\left(r_{2}, \theta_{2}\right)$ is the standing wave in the lining. $C_{01}, D_{01}$, $C_{02}$, and $D_{02}$ are undetermined complex constants. $J_{n_{2}}(x)$ is the Bessel function of the first kind with argument $x$ and order $n_{2}$. $H_{n_{2}}^{(1)}(x)$ is the Hankel function of the first kind with argument $x$ and order $n_{2}$. The wave motion in the lining is

$$
W_{0}=W_{01}\left(r_{2}, \theta_{2}\right)+W_{02}\left(r_{2}, \theta_{2}\right)
$$

The expression of the scattered wavefield generated in the loose rock circle in the polar coordinate system $\left(r_{2}, \theta_{2}\right)$ can be written as

$$
\begin{aligned}
W_{j 1}\left(r_{2}, \theta_{2}\right) & =\sum_{n_{2}=0}^{+\infty} H_{n_{2}}^{(1)}\left(k_{j} r_{2}\right)\left(C_{j 1} \cos n_{2} \theta_{2}+D_{j 1} \sin n_{2} \theta_{2}\right), \\
W_{j 2}\left(r_{2}, \theta_{2}\right) & =\sum_{n_{2}=0}^{+\infty} J_{n_{2}}\left(k_{j} r_{2}\right)\left(C_{j 2} \cos n_{2} \theta_{2}+D_{j 2} \sin n_{2} \theta_{2}\right) .
\end{aligned}
$$

Here, $W_{j 1}\left(r_{2}, \theta_{2}\right)$ is the wave propagating outward from $\mathrm{O}_{2}$ in the loose rock circle and $W_{j 2}\left(r_{2}, \theta_{2}\right)$ is the standing wave in the loose rock circle. $C_{j 1}, D_{j 1}, C_{j 2}$, and $D_{j 2}$ are undetermined complex constants. The wave motion in the loose rock circle is

$$
W_{j}=W_{j 1}\left(r_{2}, \theta_{2}\right)+W_{j 2}\left(r_{2}, \theta_{2}\right) .
$$

All the wave motions must satisfy the following boundary conditions.

1) Zero stress on lining inner surface:

$$
\tau_{r_{2} z_{2}}=\mu_{0} \frac{\partial W_{0}}{\partial r_{2}}=0 \text { at } r_{2}=a \text {. }
$$

2) Displacement and stress conditions between the lining outer surface and the surrounding rock:

$$
\begin{aligned}
W_{0} & =W_{1} \text { at } r_{2}=a, \\
\mu_{0} \frac{\partial W_{0}}{\partial r_{2}} & =\mu_{1} \frac{\partial W_{1}}{\partial r_{2}} \text { at } r_{2}=a .
\end{aligned}
$$

3) Displacement and stress conditions of the $j$ th and the $(j-1)$ th interface in the surrounding loose rock:

$$
\begin{gathered}
W_{j-1}=W_{j} \quad \text { at } \quad r_{2}=c_{j-1}, \\
\mu_{j-1} \frac{\partial W_{j-1}}{\partial r_{2}}=\mu_{j} \frac{\partial W_{j}}{\partial r_{2}} \quad \text { at } \quad r_{2}=c_{j-1} .
\end{gathered}
$$

4) Displacement and stress conditions between the outermost loose rock and the half-space:

$$
\begin{gathered}
W_{j}=W \quad \text { at } \quad r_{2}=c_{j}, \\
\mu_{j} \frac{\partial W_{j}}{\partial r_{2}}=\mu \frac{\partial W}{\partial r_{2}} \quad \text { at } \quad r_{2}=c_{j} .
\end{gathered}
$$

Since the wave functions and boundary conditions are represented in different coordinate systems, the coordinate transformation is required. With the help of Grafs addition theorem $[36,37]$ of the oblique coordinate system, coordinate transformation can be carried out between any two coordinates, and the details will not be described again.

\section{Solution to the Problem}

Substituting Eq. 9 into Eq. 13, the following can be obtained:

$$
\begin{aligned}
& C_{01} E H_{0}\left(n_{2}, a\right)+C_{02} E J_{0}\left(n_{2}, a\right)=0, \\
& D_{01} E H_{0}\left(n_{2}, a\right)+D_{02} E J_{0}\left(n_{2}, a\right)=0 .
\end{aligned}
$$

Here,

$$
\begin{array}{r}
E H_{0}\left(n_{2}, r_{2}\right)=n_{2} H_{n_{2}}^{(1)}\left(k_{0} r_{2}\right)-k_{0} r_{2} H_{n_{2}+1}^{(1)}\left(k_{0} r_{2}\right), \\
E J_{0}\left(n_{2}, r_{2}\right)=n_{2} J_{n_{2}}\left(k_{0} r_{2}\right)-k_{0} r_{2} J_{n_{2}+1}\left(k_{0} r_{2}\right) .
\end{array}
$$


Substituting Eqs. 9, 12 into Eqs. 14, 15, the following can be obtained:

$$
\begin{gathered}
C_{01} H_{n_{2}}^{(1)}\left(k_{0} b\right)+C_{02} J_{n_{2}}\left(k_{0} b\right)-C_{11} H_{n_{2}}^{(1)}\left(k_{1} b\right)-C_{12} J_{n_{2}}\left(k_{1} b\right)=0, \\
D_{01} H_{n_{2}}^{(1)}\left(k_{0} b\right)+D_{02} J_{n_{2}}\left(k_{0} b\right)-D_{11} H_{n_{2}}^{(1)}\left(k_{1} b\right)-D_{12} J_{n_{2}}\left(k_{1} b\right)=0, \\
C_{01} E H_{0}\left(n_{2}, b\right)+C_{02} E J_{0}\left(n_{2}, b\right)-\frac{\mu_{1}}{\mu_{0}}\left[C_{11} E H_{1}\left(n_{2}, b\right)\right. \\
\left.+C_{12} E J_{1}\left(n_{2}, b\right)\right]=0, \\
D_{01} E H_{0}\left(n_{2}, b\right)+D_{02} E J_{0}\left(n_{2}, b\right)-\frac{\mu_{1}}{\mu_{0}}\left[D_{11} E H_{1}\left(n_{2}, b\right)\right. \\
\left.\quad+D_{12} E J_{1}\left(n_{2}, b\right)\right]=0 .
\end{gathered}
$$

Here,

$$
\begin{array}{r}
E H_{1}\left(n_{2}, r_{2}\right)=n_{2} H_{n_{2}}^{(1)}\left(k_{1} r_{2}\right)-k_{1} r_{2} H_{n_{2}+1}^{(1)}\left(k_{1} r_{2}\right), \\
E J_{1}\left(n_{2}, r_{2}\right)=n_{2} J_{n_{2}}\left(k_{1} r_{2}\right)-k_{1} r_{2} J_{n_{2}+1}\left(k_{1} r_{2}\right) .
\end{array}
$$

Similarly, applying Eqs. 16, 17 to the $j$ th and $(j-1)$ th loose rock layers, the following can be obtained:

$$
\begin{aligned}
& C_{(j-1) 1} H_{n_{2}}^{(1)}\left(k_{j-1} c_{j-1}\right)+C_{(j-1) 2} J_{n_{2}}\left(k_{j-1} c_{j-1}\right)-C_{j 1} H_{n_{2}}^{(1)}\left(k_{j} c_{j-1}\right) \\
& \quad-C_{j 2} J_{n_{2}}\left(k_{j} c_{j-1}\right)=0 \\
& D_{(j-1) 1} H_{n_{2}}^{(1)}\left(k_{j-1} c_{j-1}\right)+D_{(j-1) 2} J_{n_{2}}\left(k_{j-1} c_{j-1}\right)-D_{j 1} H_{n_{2}}^{(1)}\left(k_{j} c_{j-1}\right) \\
& \quad-D_{j 2} J_{n_{2}}\left(k_{j} c_{j-1}\right)=0 \\
& C_{(j-1) 1} E H_{j-1}\left(n_{2}, c_{j-1}\right)+C_{(j-1) 2} E J_{j-1}\left(n_{2}, c_{j-1}\right) \\
& \quad-\frac{\mu_{j}}{\mu_{j-1}}\left[C_{j 1} E H_{j}\left(n_{2}, c_{j-1}\right)+C_{j 2} E J_{j}\left(n_{2}, c_{j-1}\right)\right]=0 \\
& D_{(j-1) 1} E H_{j-1}\left(n_{2}, c_{j-1}\right)+D_{(j-1) 2} E J_{j-1}\left(n_{2}, c_{j-1}\right) \\
& \quad-\frac{\mu_{j}}{\mu_{j-1}}\left[D_{j 1} E H_{j}\left(n_{2}, c_{j-1}\right)+D_{j 2} E J_{j}\left(n_{2}, c_{j-1}\right)\right]=0
\end{aligned}
$$

where

$$
\begin{gathered}
E H_{j-1}\left(n_{2}, r_{2}\right)=n_{2} H_{n_{2}}^{(1)}\left(k_{j-1} r_{2}\right)-k_{j-1} r_{2} H_{n_{2}+1}^{(1)}\left(k_{j-1} r_{2}\right), \\
E J_{j-1}\left(n_{2}, r_{2}\right)=n_{2} J_{n_{2}}\left(k_{j-1} r_{2}\right)-k_{j-1} r_{2} J_{n_{2}+1}\left(k_{j-1} r_{2}\right), \\
E H_{j}\left(n_{2}, r_{2}\right)=n_{2} H_{n_{2}}^{(1)}\left(k_{j} r_{2}\right)-k_{j} r_{2} H_{n_{2}+1}^{(1)}\left(k_{j} r_{2}\right) \\
E J_{j}\left(n_{2}, r_{2}\right)=n_{2} J_{n_{2}}\left(k_{j} r_{2}\right)-k_{j} r_{2} J_{n_{2}+1}\left(k_{j} r_{2}\right) .
\end{gathered}
$$

Substituting Eqs. 6, 12 into Eqs. 18, 19, respectively, the following can be obtained:

$$
\begin{aligned}
& C_{j 1} H_{n_{2}}^{(1)}\left(k_{j} c_{j}\right)+C_{j 2} J_{n_{2}}\left(k_{j} c_{j}\right)-A_{i 1} J_{n_{2}}\left(k c_{j}\right)-A_{s 4}^{*} J_{n_{2}}\left(k c_{j}\right) \\
& \quad-A_{r 3} J_{n_{2}}\left(k c_{j}\right)-A_{s 2} H_{n_{2}}^{(1)}\left(k c_{j}\right)=0, \\
& D_{j 1} H_{n_{2}}^{(1)}\left(k_{j} c_{j}\right)+D_{j 2} J_{n_{2}}\left(k_{j} c_{j}\right)-B_{s 4}^{*} J_{n_{2}}\left(k c_{j}\right)-B_{r 3} J_{n_{2}}\left(k c_{j}\right) \\
& \quad-B_{s 2} H_{n_{2}}^{(1)}\left(k c_{j}\right)=0,
\end{aligned}
$$

$$
\begin{aligned}
& C_{j 1} E H_{j}\left(n_{2}, c_{j}\right)+C_{j 2} E J_{j}\left(n_{2}, c_{j}\right) \\
& -\frac{\mu}{\mu_{j}}\left[\begin{array}{l}
A_{s 2} E H\left(n_{2}, c_{j}\right)+A_{i 1} E J\left(n_{2}, c_{j}\right) \\
+A_{s 4}^{*} E J\left(n_{2}, c_{j}\right)+A_{r 3} E J\left(n_{2}, c_{j}\right)
\end{array}\right]=0, \\
& D_{j 1} E H_{j}\left(n_{2}, c_{j}\right)+D_{j 2} E J_{j}\left(n_{2}, c_{j}\right)-\frac{\mu}{\mu_{j}}\left[B_{s 4}^{*} E J\left(n_{2}, c_{j}\right)\right. \\
& \left.\quad+B_{r 3} E J\left(n_{2}, c_{j}\right)+B_{s 2} E H\left(n_{2}, c_{j}\right)\right]=0 .
\end{aligned}
$$

Here,

$$
\begin{aligned}
& A_{s 4}^{*}=\sum_{n_{4}=0}^{+\infty}\left(A_{s 4} F_{1 H}^{+42}+B_{s 4} F_{2 H}^{+42}\right) \text { and } B_{s 4}^{*}=\sum_{n_{4}=0}^{+\infty}\left(B_{s 4} F_{1 H}^{-42}-A_{s 4} F_{2 H}^{-42}\right) \text {, } \\
& F_{1 c}^{ \pm 42}=\frac{\varepsilon_{n_{2}}}{2}\left[C_{n_{2}+n_{4}}\left(d_{24}\right) \cdot \cos \left(\frac{n_{2}-n_{4}}{2} \pi\right) \pm(-1)^{n_{4}} \cdot C_{n_{2}-n_{4}}\left(d_{24}\right)\right. \\
& \left.\cdot \cos \left(\frac{n_{2}+n_{4}}{2} \pi\right)\right] \\
& F_{2 c}^{ \pm 42}=\frac{\varepsilon_{n_{2}}}{2}\left[-C_{n_{2}+n_{4}}\left(d_{24}\right) \cdot \sin \left(\frac{n_{2}-n_{4}}{2} \pi\right) \pm(-1)^{n_{4}} \cdot C_{n_{2}-n_{4}}\left(d_{24}\right)\right. \\
& \left.\cdot \sin \left(\frac{n_{2}+n_{4}}{2} \pi\right)\right] \text {, } \\
& F_{3 c}^{ \pm 32}=\frac{\varepsilon_{n_{2}}}{2}\left[ \pm C_{n_{2}+n_{3}}\left(d_{23}\right) \cdot \cos \left(n_{2}+n_{3}\right) \gamma+(-1)^{n_{3}} \cdot C_{n_{2}-n_{3}}\left(d_{23}\right)\right. \\
& \left.\cdot \cos \left(n_{2}-n_{3}\right) \gamma\right] \text {, } \\
& F_{4 c}^{ \pm 32}=\frac{\varepsilon_{n_{2}}}{2}\left[C_{n_{2}+n_{3}}\left(d_{23}\right) \cdot \sin \left(n_{2}+n_{3}\right) \gamma \pm(-1)^{n_{3}} \cdot C_{n_{2}-n_{3}}\left(d_{23}\right)\right. \\
& \left.\cdot \sin \left(n_{2}-n_{3}\right) \gamma\right] \\
& \mathcal{E}_{n}=\left\{\begin{array}{ll}
1 & n=0 \\
2 & n>0
\end{array}, \quad \gamma=\arctan \left(\frac{2 D}{d_{12}}\right),\right. \\
& d_{23}=\sqrt{(2 D)^{2}+\left(d_{12}\right)^{2}} \text {. }
\end{aligned}
$$

In Eq. 29, the subscript $C$ is replaced with $H$ to represent the first kind of Hankel function, or the subscript $C$ is replaced with $J$ to represent the first kind of Bessel function.

Eqs. 19, 21, 22, 24, 25, and 27 constitute an infinite algebraic system of equations. Though setting the truncated number $N$, all the unknown coefficients can be obtained by solving Eqs. 19, 21, 22, 24, $\mathbf{2 5}$, and 27 together. The analytical series solution of the problem can be obtained by substituting the coefficients into the corresponding wavefields, and the corresponding stress fields can also be calculated.

\section{Dynamic Stress Concentration Factor (DSCF) of the Inner and Outer Surfaces of the Lining}

The hoop dynamic stress concentration factor (DSCF) of the inner and outer surfaces of the lining can be obtained from the normalization of the radial stress generated by the incident wave at the same point in the whole space, namely,

$$
\mathrm{DSCF}=\tau_{\theta_{2} z_{2}} / \tau_{r_{1} z_{1}}^{(i)} \quad \text { and } \quad \tau_{r_{1} z_{1}}^{(i)}=-\mu k H_{1}^{(1)}\left(k r_{1}\right) .
$$


$A^{\text {Lining stress residuals convergence }}$
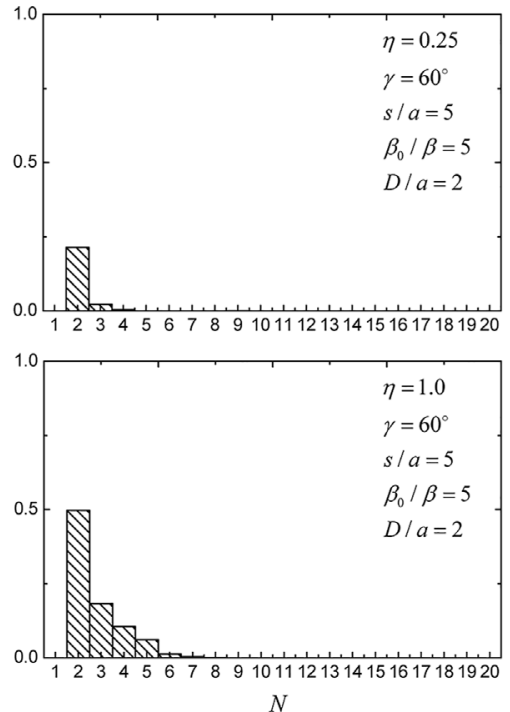

N

B

Comparison of the published results of tunnel lining without loose rock zone [33]

This paper

-.-- Ding et al
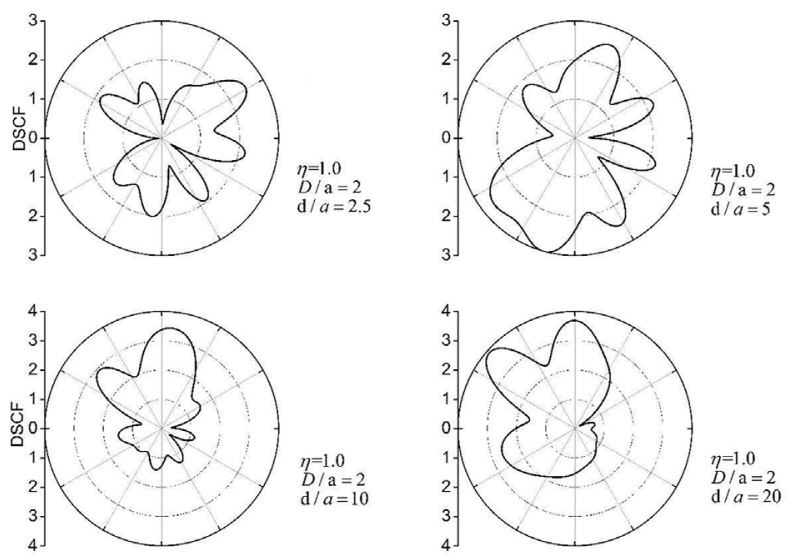

FIGURE 2 | Model verification.

Taking the outer surface of the lining as an example, the calculation formula of the DSCF is given as follows. The DSCF of the lining inner surface is similar and will not be repeated. As shown in Figure 1C, the wavefield and stress of any point can be expressed as

$$
\begin{aligned}
W_{1} & =W_{11}\left(r_{2}, \theta_{2}\right)+W_{12}\left(r_{2}, \theta_{2}\right) \\
& =\sum_{\mathrm{n} 2=0}^{+\infty}\left\{\begin{array}{l}
{\left[C_{11} H_{n_{2}}^{(1)}\left(k_{1} r_{2}\right)+C_{12} J_{n_{2}}\left(k_{1} r_{2}\right)\right] \cos n_{2} \theta_{2}} \\
+\left[D_{11} H_{n_{2}}^{(1)}\left(k_{1} r_{2}\right)+D_{12} J_{n_{2}}\left(k_{1} r_{2}\right)\right] \sin n_{2} \theta_{2}
\end{array}\right\}, \\
\sigma_{\theta_{2} z_{2}} & =\frac{\mu_{1}}{b} \frac{\partial W_{1}}{\partial \theta_{2}} \\
& =\frac{\mu_{1}}{b} \sum_{n 2=0}^{+\infty} n_{2} \times\left\{\begin{array}{l}
{\left[D_{11} H_{n_{2}}^{(1)}\left(k_{1} r_{2}\right)+D_{12} J_{n_{2}}\left(k_{1} r_{2}\right)\right] \cos n_{2} \theta_{2}} \\
-\left[C_{11} H_{n_{2}}^{(1)}\left(k_{1} r_{2}\right)+C_{12} J_{n_{2}}\left(k_{1} r_{2}\right)\right] \sin n_{2} \theta_{2}
\end{array}\right\},
\end{aligned}
$$

where $r_{1}=b^{2}+d_{12}^{2}-2 b \times d_{12} \times \cos \theta_{2}$. Then, the DSCF of the outer surface of the lining can be obtained as

$$
\begin{aligned}
\mathrm{DSCF} & =\tau_{\theta_{2} z_{2}} / \tau_{r_{1} z_{1}}^{(i)} \\
& =-\frac{\mu_{1}}{\mu} \frac{1}{k b H_{1}^{(1)}\left(k r_{1}\right)} \sum_{n 2=0}^{+\infty} n_{2} \times\left\{\begin{array}{l}
{\left[D_{11} H_{n_{2}}^{(1)}\left(k_{1} r_{2}\right)+D_{12} J_{n_{2}}\left(k_{1} r_{2}\right)\right] \cos n_{2} \theta_{2}} \\
-\left[C_{11} H_{n_{2}}^{(1)}\left(k_{1} r_{2}\right)+C_{12} J_{n_{2}}\left(k_{1} r_{2}\right)\right] \sin n_{2} \theta_{2}
\end{array}\right\} .
\end{aligned}
$$

\section{SOLUTION VERIFICATION}

The dimensionless frequency $\eta$, which is expressed in terms of the tunnel radius $a$ and the wave velocity $\beta$, is defined as [38]

$$
\eta=\frac{2 a}{\lambda}=\frac{\omega a}{\pi \beta},
$$


$D / a=2$

Case 1
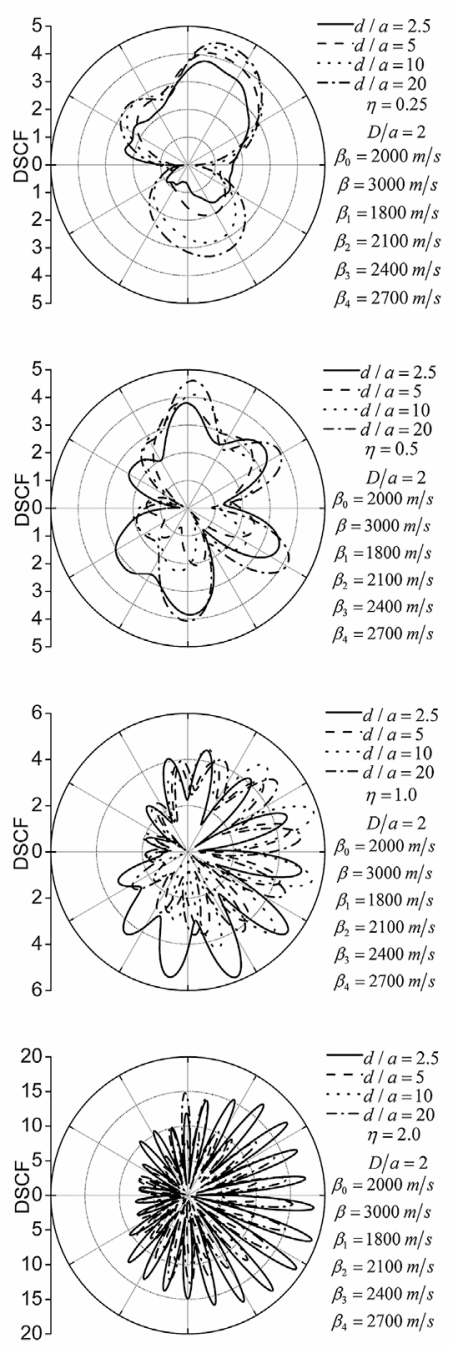

Case 2
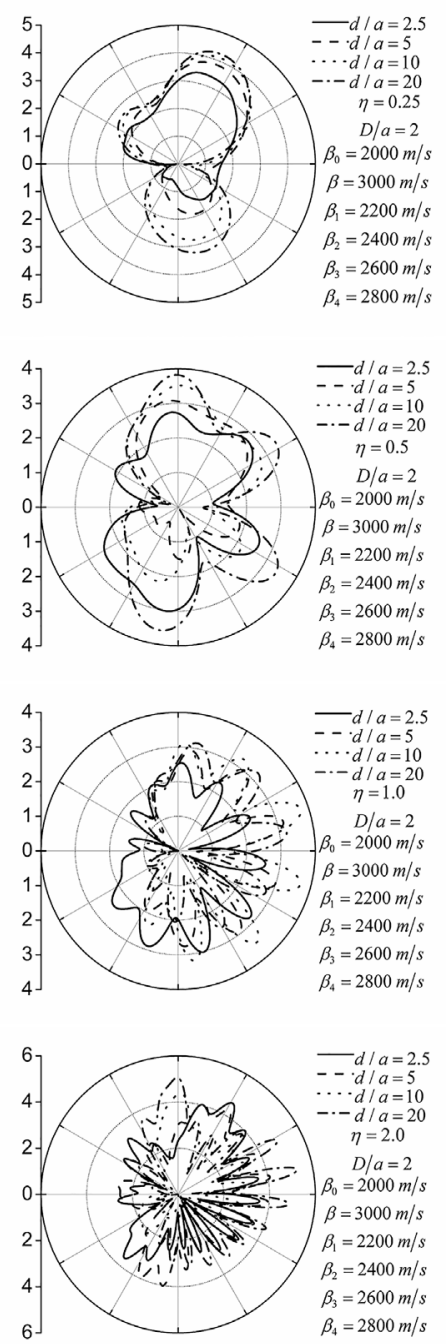

Case 3
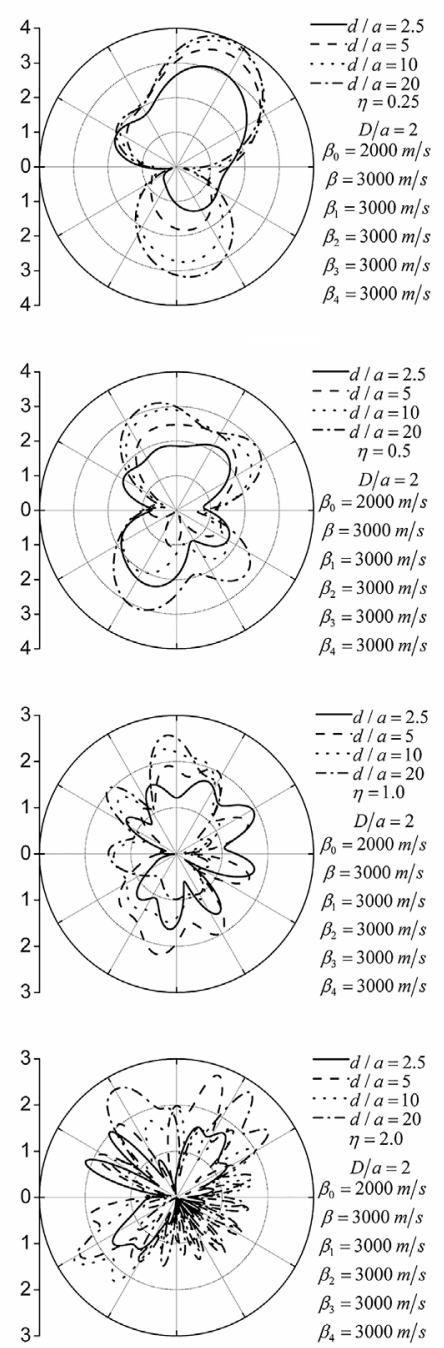

FIGURE 3 |DSCF of the lining outer surface for the lining burial depth $D / a=2$. Different lines (solid, dash, dot, and dash-dot lines), respectively, correspond to the wave source-lining distances $\mathrm{d} / \mathrm{a}=2.5,5,10$, and 20. Rows $1-4$ correspond to the dimensionless frequencies of the incident cylindrical SH-wave $\eta=0.25,0.5,1.0$, and 2.0. Columns $1-3$ correspond to the three cases.

where $2 a$ is the tunnel diameter and $\lambda$ is the wavelength of the shear waves in the half-space.

\section{Precision Variation With the Truncated Number $\boldsymbol{N}$}

Figure 2A shows the convergence of lining stress residual at the outer surface under four different dimensionless frequencies $\eta=$ $0.25,0.5,1.0$, and 2.0 , when $d_{12} / b=5$ and tunnel buried depth $D /$ $a=2$. For different incident wave frequencies, with the increase of truncation terms $N$, the error gradually approaches zero, which proves that the series solution in this paper can obtain a result that meets the accuracy.

\section{Comparison With the Published Results}

Taking the surrounding loose rock of four layers as an example, Figure 2B shows the comparison between our results and the published results [32] (tunnel lining without loose rock zone) when $d / a=2.5,5,10$, and $20, \beta_{0} / \beta=1, \rho_{0} / \rho=1, \beta_{1}=\beta_{2}=\beta_{3}$ $=\beta_{4}$, and $\rho_{1}=\rho_{2}=\rho_{3}=\rho_{4}$. The figure demonstrates that our results agree well with the published results, indicating the correctness of our solution.

\section{RESULTS AND ANALYSIS}

Figure 3 and Figure 4 demonstrate the results of the circumferential DSCF of the lining outer surface under 
$D / a=5$

Case 1
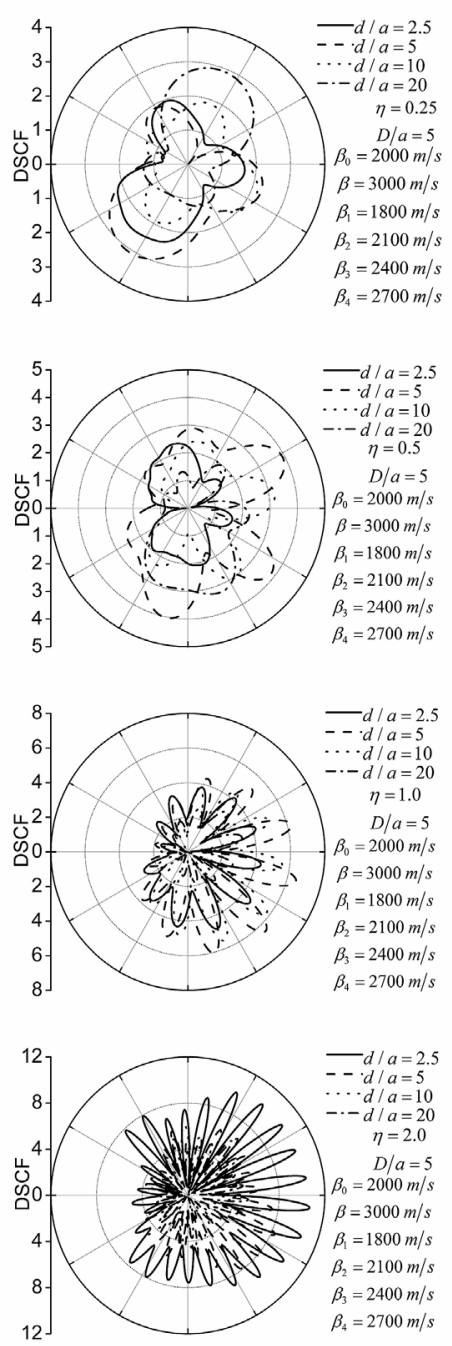

Case 2
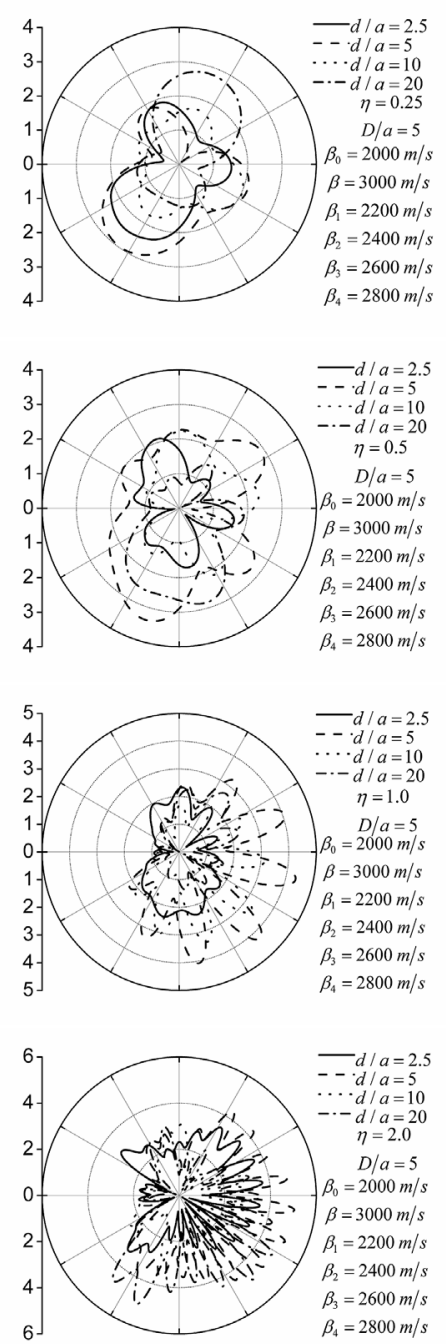

Case 3
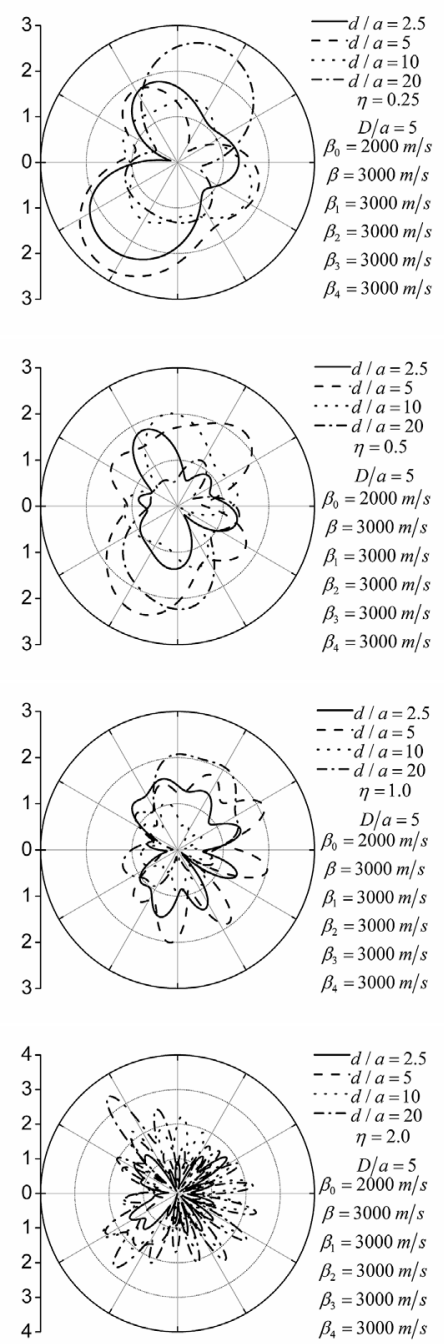

FIGURE 4 | The same as Figure 3 but for the lining burial depth $D / a=5$.

different lining burial depths $D / a=2$ and 5. The calculation parameters are as follows. For the lining, the shear wave velocity is $\beta_{0}=2000 \mathrm{~m} / \mathrm{s}$, Poisson's ratio is $\nu_{0}=0.2$, and mass density $\rho_{0}=$ $2,500 \mathrm{~kg} / \mathrm{m}^{3}$; for the half-space, the shear wave velocity is $\beta=$ $3,000 \mathrm{~m} / \mathrm{s}$, Poisson's ratio is $v=0.25$, and mass density $\rho=$ $2,750 \mathrm{~kg} / \mathrm{m}^{3}$. The surrounding loose rock zone is assumed to be divided into four layers, and the properties of the loose rock zone are discussed in three cases as follows:

Case 1: $\beta_{1}=1800 \mathrm{~m} / \mathrm{s}, \beta_{2}=2100 \mathrm{~m} / \mathrm{s}, \beta_{3}=2400 \mathrm{~m} / \mathrm{s}, \beta_{4}=$ $2700 \mathrm{~m} / \mathrm{s}$.

Case 2: $\beta_{1}=2200 \mathrm{~m} / \mathrm{s}, \beta_{2}=2400 \mathrm{~m} / \mathrm{s}, \beta_{3}=2600 \mathrm{~m} / \mathrm{s}, \beta_{4}=$ $2800 \mathrm{~m} / \mathrm{s}$.

Case 3: $\beta_{1}=3000 \mathrm{~m} / \mathrm{s}, \beta_{2}=3000 \mathrm{~m} / \mathrm{s}, \beta_{3}=3000 \mathrm{~m} / \mathrm{s}, \beta_{4}=$ $3000 \mathrm{~m} / \mathrm{s}$.
Case 1 represents a large degree of surrounding rock loosening around the lining, Case 2 represents a moderate degree of surrounding rock loosening, and Case 3 corresponds to no surrounding rock loosening.

The DSCF of the lining outer surface at different incident frequencies $(\eta=0.25,0.5,1.0$, and 2.0$)$ is shown in Figure 3 and Figure 4. It can be found that the amplitude of the DSCF changes gently along the circumference of the lining when the incident wave has a relatively lower frequency $(\eta=$ $0.25)$. With the increase of the frequency of the incident wave, the amplitudes of the DSCF change dramatically along the lining circumference. This indicates that, with the increase of incident wave frequency, the refraction and scattering of incident waves in the lining and surrounding loose rock zone are intensified, which leads to the intensification of 
dynamic stress concentration on the outer surface of the lining.

By comparing and analyzing the DSCF amplitude under different wave source-lining distances $(d / a=2.5,5,10$, and $20)$, it can be found that the distance $(d / a)$ between the wave source and the lining has a significant influence on the DSCF. Particularly, an interesting phenomenon can be observed, that is, with the increase of the distance between the wave source and the cavity, the DSCF gradually increases, which is obviously different from that of the plane $\mathrm{SH}$-wave. The reasons for this significant difference can be explained as follows. In the case of cylindrical $\mathrm{SH}$-wave incidence, the denominator (stress amplitude generated by the incident cylindrical $\mathrm{SH}$-wave in the free-field) in the normalization formula (Eq. 34) of the DSCF is attenuated. However, for the incident plane $\mathrm{SH}$-wave, the denominator (stress amplitude caused by the plane $\mathrm{SH}$-wave in the freefield) is constant.

The lining buried depth $(D / a)$ also has a significant effect on the DSCF. It can be seen from Figure 3 and Figure 4 that when the lining buried depth is larger $(D / a)$, the DSCF of the lining outer surface decreases to a certain extent, and the distribution of the DSCF changes dramatically. This shows that the ground surface has an important influence on the DSCF. At the same time, it can be seen that when the lining buried depth of the lining is large, the influence of a low-frequency wave is larger and that of a high-frequency wave is relatively small due to the large distance between the lining and the ground surface, which is particularly noteworthy.

The three surrounding rock case analyses include the case of no loosening of surrounding rock (Case 3). Currently, the dynamic stress concentration factor (DSCF) is relatively small, that is, the stress concentration degree is smaller than the result of the surrounding loosening rock case. In the other two cases, the surrounding rock stiffness changes linearly, and the DSCF changes greatly, indicating that the dynamic stress concentration is more obvious.

\section{CONCLUSION}

In this paper, the closed-form series solution of cylindrical SHwave scattering by surrounding rock in a uniform half-space is obtained by using the wave function expansion method. Considering that blasting will inevitably loosen the surrounding rock around the tunnel lining in practical engineering, we analyze the influence of the frequency of the incident cylindrical $\mathrm{SH}$-wave, the distance between the wave source and the lining, the lining buried depth, and the properties of the surrounding loose rock zone on the dynamic

\section{REFERENCES}

1. Trifunac MD. Scattering of Plane SH Wave by a Semi-cylindrical canyon. Earthquake Eng Struct Dyn (1973) 1:267-81. doi:10.1002/ eqe. 4290010307 stress concentration of the tunnel lining, based on this series solution. The conclusions and findings are as follows:

1) Generally speaking, the incident wave with high frequency always makes the dynamic stress concentration of the tunnel lining obvious. The variation of the dynamic stress concentration factor (DSCF) curve of the lining outer surface is complex and violent, and the distribution is not uniform.

2) With the increase of the distance between the wave source and the tunnel, the stress around the tunnel lining decreases, but the dynamic stress concentration factor around the tunnel lining does not decrease significantly but occasionally increases. This is because in the calculation formula of the normalized dynamic stress concentration factor, the denominator decreases faster than the hoop stress of the lining.

3) In general, the amplitude of hoop stress in the tunnel lining decreases with the increase of lining buried depth. This is enough to show that the ground surface has a great influence on the stress concentration of the tunnel lining.

4) When other conditions are the same, the stress concentration of the surrounding loose rock zone is more obvious than that without loose rock zone. When the property of the surrounding rock (namely, shear wave velocity) changes more, the amplitude of the stress concentration factor is larger, that is, the stress concentration is more significant.

\section{DATA AVAILABILITY STATEMENT}

The raw data supporting the conclusions of this article will be made available by the authors, without undue reservation.

\section{AUTHOR CONTRIBUTIONS}

LJ and ZZ conceptualized the study. HS and LJ performed mathematical derivation and ran the computer program. LJ validated the results, wrote the original draft, and reviewed and edited the paper. SW curated the data. All authors read and agreed to publish this manuscript.

\section{FUNDING}

This work was supported by the National Natural Science Foundation of China (Grant Nos. 51808290, U2039208, and 41902282).

\footnotetext{
2. Trifunac MD. Surface Motion of a Semi-cylindrical Alluvial valley for Incident Plane SH Waves. Bull Seismology Soc America (1971) 61:1755-70. doi:10.1785/ bssa0610061755

3. Lee VW, Trifunac MD. Response of Tunnels to Incident SH-Waves. $J$ Engrg Mech Div.ASCE (1979) 105(4):643-59. doi:10.1061/ jmcea3.0002511
} 
4. Zhang N, Chen X, Gao Y, Dai D. Analytical Solution to Scattering of SH Waves by a Circular Lined Tunnel Embedded in a Semi-circular Alluvial valley in an Elastic Half-Space. Tunnelling Underground Space Tech (2020) 106:103615. doi:10.1016/j.tust.2020.103615

5. Tsaur DH. Exact Scattering and Diffraction of Anti-plane Shear Waves by a Vertical Edge Crack. Geophys J Int (2010) 181(3):1655-64. doi:10.1111/j.1365246x.2010.04577.x

6. Gao Y, Zhang N, Li D, Liu H, Cai Y, Wu Y. Effects of Topographic Amplification Induced by a U-Shaped canyon on Seismic Waves. Bull Seismological Soc America (2012) 102(4):1748-63. doi:10.1785/0120110306

7. Zhang N, Gao Y, Cai Y, Li D, Wu Y. Scattering of SH Waves Induced by a Nonsymmetrical V-Shaped canyon. Geophys J Int (2012) 191(1):243-56. doi:10.1111/j.1365-246x.2012.05604.x

8. Jin L, Liang J. 2D Dynamic Structure-canyon-structure Interaction for the Buildings along the Urban River-canyon I: Incident SH-Waves in Homogenous Half-Space. J Earthquake Eng (2020) 1-19. doi:10.1080/13632469.2020.1785587

9. Jin L, Tang G, Liang J. Dynamic Soil-Structure-Equipment Interaction (I): Closedform Analytical Solution for Incident Plane SH Wave Based on Rigid Foundation Model. J Earthquake Eng (2019) 1-17. doi:10.1080/13632469.2019.1633972

10. Jin L, Zhou W, Liang J, Huang Y. Dynamic Soil-Structure-Equipment Interaction (II): Closed-form Analytical Solution for Incident Plane SHWave Based on Flexible Foundation Model. J Earthquake Eng (2020) 1-26. doi:10.1080/13632469.2020.1840458

11. Lee VW, Liu W-Y. Two-dimensional Scattering and Diffraction of P- and SVWaves Around a Semi-circular canyon in an Elastic Half-Space: an Analytic Solution via a Stress-free Wave Function. Soil Dyn Earthquake Eng (2014) 63(63):110-9. doi:10.1016/j.soildyn.2014.02.005

12. Lee VW, Zhu G. A Note on Three-Dimensional Scattering and Diffraction by a Hemispherical canyon-I: Vertically Incident Plane P-Wave. Soil Dyn Earthquake Eng (2014) 61-62(2):197-211. doi:10.1016/j.soildyn.2014.02.010

13. Lan H, Zhang Z. Three-dimensional Wave-Field Simulation in Heterogeneous Transversely Isotropic Medium with Irregular Free Surface. Bull Seismological Soc America (2011) 101(3):1354-70. doi:10.1785/0120100194

14. Zhang W, Chen X. Traction Image Method for Irregular Free Surface Boundaries in Finite Difference Seismic Wave Simulation. Geophys J Int (2006) 167(1):337-53. doi:10.1111/j.1365-246x.2006.03113.x

15. Zhou H, Chen X. A New Approach to Simulate Scattering of SH Waves by an Irregular Topography. Geophys J Int (2006) 164(2):449-59. doi:10.1111/j.1365246x.2005.02670.x

16. Liang J, Liu Z. Diffraction of Plane P Waves by a canyon of Arbitrary Shape in Poroelastic Half-Space (I): Formulation. Earthq Sci (2009) 22(3):215-22. doi:10.1007/s11589-009-0215-y

17. Wong HL. Effect of Surface Topography on the Diffraction of P, SV, and Rayleigh Waves. Bull Seismology Soc America (1982) 72(4):1167-83.

18. Chen J-T, Chen P-Y, Chen C-T. Surface Motion of Multiple Alluvial Valleys for Incident Plane Sh-Waves by Using a Semi-analytical Approach. Soil Dyn Earthquake Eng (2008) 28(1):58-72. doi:10.1016/j.soildyn.2007.04.001

19. Lee VW, Wu X. Application of the Weighted Residual Method to Diffraction by 2-D Canyons of Arbitrary Shape: I. Incident SH Waves. Soil Dyn Earthquake Eng (1994) 13(5):355-64. doi:10.1016/0267-7261(94)90026-4

20. Lee VW, Brandow HP. Weighted Residual Method for Diffraction of Plane P-Waves in a 2D Elastic Half-Space Revisited: on an Almost Circular ArbitraryShaped canyon. J Earthquakes (2015) 2015:1-21. doi:10.1155/2015/543128

21. Lee VW, Wu X. Application of the Weighted Residual Method to Diffraction by 2-D Canyons of Arbitrary Shape: II. Incident P, SV and Rayleigh Waves. Soil Dyn Earthquake Eng (1994) 13(5):365-75. doi:10.1016/0267-7261(94)90027-2

22. Luco JE, Wong HL, De Barros FCP. Three-dimensional Response of a Cylindrical canyon in a Layered Half-Space. Earthquake Engng Struct Dyn (1990) 19:799-817. doi:10.1002/eqe.4290190603

23. Sanchez-Sesma FJ, Campillo M. Diffraction of P, SV, and Rayleigh Waves by Topographic Features: a Boundary Integral Formulation. Bull Seismology Soc America (1991) 81(6):2234-53.

24. Vogt RF, Wolf JP, Bachmann H. Wave Scattering by a canyon of Arbitrary Shape in a Layered Half-Space. Earthquake Engng Struct Dyn (1988) 16: 803-12. doi:10.1002/eqe. 4290160603
25. Kawase H. Time-domain Response of a Semi-circular canyon for Incident SV, $\mathrm{P}$, and Rayleigh Waves Calculated by the Discrete Wavenumber Boundary Element Method. Bull Seismology Soc America (1988) 78(4):1415-37. doi:10.1785/bssa0780041415

26. Liang JW, You HB, Lee VW. Scattering of SV Waves by a canyon in a FluidSaturated, Poroelastic Layered Half-Space, Modeled Using the Indirect Boundary Element Method. Soil Dyn Earthquake Eng (2006) 26(7):611-25. doi:10.1016/j.soildyn.2006.01.012

27. Ba Z, Yin X. Wave Scattering of Complex Local Site in a Layered Half-Space by Using a Multidomain IBEM: Incident Plane SH Waves. Geophys J Int (2016) 205:1382-405. doi:10.1093/gji/ggw090

28. Ba Z, Lee VW, Liang J, Yan Y. Scattering of Plane qP- and qSV-Waves by a canyon in a Multi-Layered Transversely Isotropic Half-Space. Soil Dyn Earthquake Eng (2017) 98:120-40. doi:10.1016/j.soildyn.2017.04.005

29. Ba Z, Fu Z, Liu Z, Sang Q. A 2.5D IBEM to Investigate the 3D Seismic Response of 2D Topographies in a Multi-Layered Transversely Isotropic Half-Space. Eng Anal Boundary Elem (2020) 113:382-401. doi:10.1016/j.enganabound.2020.01.019

30. Sanchez-Sesma FJ, Palencia VJ, Luzon F. Estimation of Local Site Effects during Earthquakes: an Overview. ISET J Earthquake Tech (2002) 39:167-93.

31. Liu G, Chen H, Liu D, Khoo BC. Surface Motion of a Half-Space with Triangular and Semicircular hills under Incident SH Waves. Bull Seismological Soc America (2010) 100(3):1306-19. doi:10.1785/ 0120090273

32. Liang JW, Ding M, Du J. Diffraction of Cylindrical SH Waves Around Circular Lined Cavity: Analytical Solution. J Earthquake Eng Eng Vibration (2013) 33(1):1-7.

33. Li Y. Scattering of Cylindrical SH Waves by Underground, Circular Cavity. Dissertation. Tianjin: Tianjin University (2004). (in Chinese).

34. Zhang M. Scattering of Cylindrical SH Waves by Underground Circular Inclusion and Lined Tunnels. Dissertation. Tianjin: Tianjin University (2007). (in Chinese).

35. Xu Y, Liang JW, Liu ZX. Diffraction of Rayleigh Waves Around a Circular Cavity in Poro-Elastic Half-Space. Rock Soil Mech (2017) 38(8):2412-24. (in Chinese).

36. Jin L, Liang J. Dynamic Soil-Structure Interaction with a Flexible Foundation Embedded in a Half-Space: Closed-form Analytical Solution for Incident Plane SH Waves. J Earthquake Eng (2019) 25:1565-89. doi:10.1080/ 13632469.2019.1586802

37. Abramowitz M, Stegun IA. Handbook of Mathematical Functions with Formulas, Graphs, and Mathematical Tables. New York: Dover (1972).

38. Trifunac MD. Dynamic Interaction of a Shear wall with the Soil for Incident Plane SH Waves. Bull Seismology Soc America (1972) 62:62-83. doi:10.1785/ bssa0620010063

39. Bhatti M, Lu D. Analytical Study of the Head-On Collision Process between Hydroelastic Solitary Waves in the Presence of a Uniform Current. Symmetry (2019) 11(3):333. doi:10.3390/sym11030333

40. Bhatti MM, Lu DQ. An Application of Nwogu's Boussinesq Model to Analyze the Head-On Collision Process between Hydroelastic Solitary Waves. Open Phys 17(1):177-91. doi:10.1515/phys-2019-0018

Conflict of Interest: The authors declare that the research was conducted in the absence of any commercial or financial relationships that could be construed as a potential conflict of interest.

Publisher's Note: All claims expressed in this article are solely those of the authors and do not necessarily represent those of their affiliated organizations, or those of the publisher, the editors, and the reviewers. Any product that may be evaluated in this article, or claim that may be made by its manufacturer, is not guaranteed or endorsed by the publisher.

Copyright (c) 2021 Jin, Sun, Wang and Zhou. This is an open-access article distributed under the terms of the Creative Commons Attribution License (CC $B Y)$. The use, distribution or reproduction in other forums is permitted, provided the original author(s) and the copyright owner(s) are credited and that the original publication in this journal is cited, in accordance with accepted academic practice. No use, distribution or reproduction is permitted which does not comply with these terms. 